\title{
Fertility of frozen-thawed stallion semen cannot be predicted by the currently used laboratory methods P Kuisma1 ${ }^{1}$ M Andersson'1, E Koskinen ${ }^{2}$ and T Katila*1
}

\author{
Address: ${ }^{1}$ Department of Clinical Veterinary Sciences, University of Helsinki, 04920 Saarentaus, Finland and ${ }^{2}$ Department of Animal Sciences, \\ University of Helsinki, PL 28, 00014 Helsingin yliopisto, Finland \\ Email: P Kuisma - paivi.kuisma@iki.fi; M Andersson - magnus.andersson@helsinki.fi; E Koskinen - erkki.koskinen@mmm.fi; \\ T Katila* - terttu.katila@helsinki.fi \\ * Corresponding author
}

Published: 17 August 2006

Acta Veterinaria Scandinavica 2006, 48:14 doi:10.1I86/I75I-0I47-48-14

This article is available from: http://www.actavetscand.com/content/48/1/14

(C) 2006 Kuisma et al; licensee BioMed Central Ltd.

This is an Open Access article distributed under the terms of the Creative Commons Attribution License (http://creativecommons.org/licenses/by/2.0), which permits unrestricted use, distribution, and reproduction in any medium, provided the original work is properly cited.
Received: 15 June 2006

Accepted: 17 August 2006

\begin{abstract}
The aim of the project was to use current simple and practical laboratory tests and compare results with the foaling rates of mares inseminated with commercially produced frozen semen. In Exp. I, semen was tested from 27 and in Exp. 2 from 23 stallions; 19 stallions participated in both experiments. The mean number of mares per stallion in both experiments was 37 ( $\min .7$, max. 121). Sperm morphology was assessed and bacterial culture performed once per stallion. In Exp. I, progressive motility after $0, \mathrm{I}, 2,3$, and $4 \mathrm{~h}$ of incubation using light microscopy, motility characteristics measured with an automatic sperm analyzer, plasma membrane integrity using carboxyfluorescein diacetate/propidium iodide (CFDA/PI) staining and light microscopy, plasma membrane integrity using $\mathrm{Pl}$ staining and a fluorometer, plasma membrane integrity using a resazurin reduction test, and sperm concentration were evaluated. In Exp. 2, the same tests as in Exp. I and a hypo-osmotic swelling test (HOST) using both light microscopy and a fluorometer were performed immediately after thawing and after a 3-h incubation. Statistical analysis was done separately to all stallions and to those having $\geq 20$ mares; in addition, stallions with foaling rates < 60 or $\geq 60 \%$ were compared. In Exp. I, progressive motility for all stallions after a 2 - 4-h incubation correlated with the foaling rate (correlation coefficients $0.39-0.5 \mathrm{I}),(\mathrm{p}<0.05)$. In stallions with $>$ 20 mares, the artificial insemination dose showed a correlation coefficient of $-0.58(p<0.05)$. In Exp. 2, the HOST immediately after thawing showed a negative correlation with foaling rate $(\mathrm{p}<$ 0.05 ). No single test was consistently reliable for predicting the fertilizing capacity of semen, since the 2 experiments yielded conflicting results, although the same stallions sometimes participated in both. This shows the difficulty of frozen semen quality control in commercially produced stallion semen, and on the other hand, the difficulty of conducting fertility trials in horses.
\end{abstract}

\section{Background}

In many countries, artificial insemination (AI) has superseded natural mating as a breeding method for mares. Use of frozen semen, however, has not gained widespread use in horses, due to low pregnancy rates. In addition to semen quality, many other factors affect the outcome of
AI, including the handling and freezing methods of semen, AI dose, timing of AI and management and fertility of the mares [1]. There is considerable variation between individual stallions in how their semen survives freezing and thawing. Otherwise fertile stallions can produce semen that results in very poor post-thaw pregnancy 
rates [2]. Tischner [3] estimated that approx. $20 \%$ of stallions are "good freezers", another $20 \%$ are "bad freezers", and the majority of stallions, $60 \%$, produce semen that is affected adversely, but may be freezable using certain techniques. Unlike bulls, stallions are not selected for breeding on the basis of fertility or semen freezability [1]. Therefore, not much progress is to be expected in the use of frozen stallion semen. For prediction of fertility and for improving freezing methods, it is important to develop reliable techniques to assess the quality of semen in vitro. Many methods exist and are used, but not many studies have examined or showed the connection between laboratory test results and fertility of frozen-thawed stallion semen [4], [5]. The relationship between motility, the most frequently used test in horses, and fertility is far from clear [6], [7] and particularly for frozen semen it is not an exact measure of fertilizing potential [8]. In Malmgren's review [9], conflicting correlations were reported between morphology - another commonly used test - and fertility of fresh stallion semen. It is often assumed that the condition of spermatozoa surviving after cryopreservation would be similar to the pre-freeze state. There is evidence that also the survivors have been affected [10]. Therefore, assessment and methods of examination applied for fresh semen may not be as useful for frozen semen.

Amann [11] stated that establishing a correlation between different attributes of semen and fertility is not sufficient. The goal is to develop laboratory tests that are predictive of fertility, which is not an easy task to achieve, particularly in horses. To determine if a laboratory test is correlated with fertility, one must have specific, precise, and accurate laboratory tests and precise and accurate fertility data from an adequate number of females. Tests of several independent parameters should be made [11-13,7]. Graham [14] listed several attributes that a sperm must possess to fertilize an oocyte, including motility, normal morphology, sufficient metabolism for energy production, and membrane integrity. Measurement of only a single attribute will fail to detect sperm defective in a different attribute and will overestimate the number of fertile sperm in the sample.

Obtaining good fertility data is difficult in horses. The number of mares and stallions used is too small, too few mares are inseminated at the appropriate time using adequate AI doses, too few semen samples are evaluated in an appropriate manner from each male, and the fertility data of mares is inaccurate [11].

Sperm membranes are particularly vulnerable during freezing [10]. This suggests that tests evaluating sperm membrane integrity should be used in the evaluation of frozen semen. On the other hand, spermatozoa that have survived freezing and thawing may be a selected subpop- ulation, which has unusually stable membranes. These membranes may also be unresponsive to physiological stimuli. If this is the case, then cryopreservation process may select viable, but relatively infertile sperm [10]. Membranes of cryopreserved spermatozoa are less able to withstand osmotic stress than fresh spermatozoa [15]. Velocity (curvilinear and mean path velocities) and linearity of cryopreserved spermatozoa are generally reduced [10]. A commonly used selection criterion in commercial stallion semen production is post-thaw progressive motility of $\geq$ $30-35 \%$.

The aim of the present study was to use economically feasible and simple laboratory tests and correlate them with the foaling rates of mares. The pregnancy rates per cycle would have better reflected fertility [16], but they were not available from all mares. The aim of the study was also to analyze the overall quality of commercially produced semen doses.

\section{Materials and methods}

Results of frozen semen evaluation tests and foaling rates of mares were compared in 2 experiments. In the first experiment, semen of 27 stallions was tested and in the second experiment semen of 23 stallions; 19 stallions participated in both experiments. Only stallions having foaling data from at least 7 mares were included; the data were also analyzed separately for stallions having $\geq 20$ mares.

\section{Frozen semen}

\section{First experiment}

Semen straws, frozen between 1988 and 1997, were available from 27 commercial stallions from Sweden (22), Finland (2), Italy (2), and the USA (1). Twelve of the stallions were American Standardbreds and 15 others represented various breeds of riding horses. Semen from one stallion was frozen in 5 - $\mathrm{mL}$ straws, from 22 in $2.5-\mathrm{mL}$ straws and from 4 stallions in $0.5-\mathrm{mL}$ straws. The foaling data originated from Finland and Sweden from 1989 to 1998. The mean number of mares per stallion was 37 ( $\min .7$, max. 121). The average foaling rate was $56 \%$ (min. 0 , max. $86 \%$ ). Twelve stallions had foaling rates $>60 \%$ and 15 had foaling rates $<60 \%$.

\section{Second experiment}

Semen straws, frozen between 1988 and 1998, were available from 23 commercial stallions from Sweden (18), Finland (3), Italy (1) and Germany (1). Semen from 18 stallions was frozen in $2.5-\mathrm{mL}$ straws and 5 in $0.5-\mathrm{mL}$ straws. Seven stallions were American Standardbreds and 16 were various breeds of riding horses. The foaling data originated from Finland and Sweden from 1989 to 1999. The average number of mares per stallion was 37 (min. 7, max. 121). The mean foaling rate was $60 \%(\min .11 \%$, 
max. $86 \%$ ). Fourteen stallions had foaling rates of $>60 \%$ and nine $<60 \%$.

\section{Experiments}

In the first experiment, the semen evaluations were performed once immediately after thawing, but motility assessment using light microscopy was continued for $4 \mathrm{~h}$. Since the incubation appeared to differentiate sperm more readily than examination immediately after thawing, all tests were carried out 0 and $3 \mathrm{~h}$ after thawing in the second experiment. Bacterial culture was performed only in the first experiment and the hypo-osmotic swelling test (HOST) only in the second experiment. The HOST was included in the evaluation tools due to promising results in stallions $[17,18]$. The morphology was assessed from frozen-thawed spermatozoa once per stallion.

\section{Thawing and incubation}

The $0.5-\mathrm{mL}$ straws were thawed at $37^{\circ} \mathrm{C}$ for $30 \mathrm{sec}$, the 2.5-mL straws at $50^{\circ} \mathrm{C}$ for 40 and the 5-mL straws for 45 sec. The semen concentration was measured in a Bürker counting chamber, and the total number of spermatozoa per straw calculated. An insemination dose was one straw when the 2.5- or 5-mL straws were used and from 1 to 10 straws for the $0.5-\mathrm{mL}$ straws. The semen was extended with a warm $\left(+30^{\circ} \mathrm{C}\right)$ skim milk extender [19] to a concentration of $20-30 \times 10^{6}$ spermatozoa $/ \mathrm{mL}$.

The sample for the longevity test was prepared by placing $0.5 \mathrm{~mL}$ of extended semen into a 3 -mL vial enclosed with a cap. The sample was kept in a water bath at $37^{\circ} \mathrm{C}$ for 4 h (Exp. 1) or $3 \mathrm{~h}$ (Exp. 2). The total and progressive motility and velocity were evaluated by light microscope every hour (Exp. 1) or after 3 h (Exp. 2).

\section{Motility}

The post-thaw motility was evaluated with a light microscope for the percentage of progressively motile spermatozoa, total motility percentage and a velocity score (from 1 to 3). Motility parameters were measured with an automatic sperm analyzer (Hamilton Thorn Motility Analyzer, HTM-S, version 7.2, Hamilton Thorne Research, Beverly, MA, USA) using video taping [20]. A 7- $\mu \mathrm{L}$ semen sample was placed into a Makler chamber at a temperature of $37.1^{\circ} \mathrm{C} ; 2$ chambers were prepared from the same sample. The chamber was placed on the thermostatically controlled stage of the motility analyzer and video recordings made as described by Varner et al. [20]. When the videotapes were analyzed the analyzer settings were: frames at frame rate $20-25 / \mathrm{sec}$, minimum contrast 8 , minimum size 6 , low/high size gates $0.6-1.5$, low/high intensity gates $0.6-1.5$, motile head size 16 , non- motile intensity 371, medium VAP (average path velocity) value 30, low VAP value 10, slow cells not motile, and threshold straightness 60 . The videotapes were analyzed for the level of total (TMOT) and progressive motility (PROG), VAP and percentage of rapid sperm (RAP).

\section{Plasma membrane integrity}

Plasma membrane integrity was evaluated after thawing, using 3 methods in Exp. 1 and 5 methods in Exp. 2: 1) carboxyfluorescein diacetate/propidium iodide (CFDA/PI) staining and counting of cells with a fluorescence microscope, 2) PI staining and measurement with a fluorometer (Fluoroscan Ascent, Thermo Electron Inc., Milford, MA, USA), 3) resazurin reduction test with a fluorometer, 4) HOST and counting cells with a microscope (only in Exp. 2) and 5) HOST using a fluorometer (only in Exp. 2). In Exp. 1, the tests were performed once immediately after thawing, while in Exp. 2 they were repeated after a 3-h incubation.

For evaluation of plasma membrane integrity with CFDA/ PI staining, the semen was extended with a skim milk extender [19] to a concentration of $50 \times 10^{6}$ spermatozoa/ $\mathrm{mL}$. Aliquots of $20 \mu \mathrm{L}$ of CFDA stock solution consisting of $0.46 \mathrm{mg}$ CFDA in $1 \mathrm{~mL}$ of DMSO (dimethylsulpfoxide) and $10 \mu \mathrm{l}$ of PI stock solution ( $0.5 \mathrm{mg}$ PI in $1 \mathrm{~mL}$ of $0.9 \%$ $\mathrm{NaCl}$ solution) were taken, mixed with $950 \mu \mathrm{l}$ of semen, and incubated for $8 \mathrm{~min}$ at $30^{\circ} \mathrm{C} \mathrm{[21].} \mathrm{A} \mathrm{5- \mu L} \mathrm{drop} \mathrm{was}$ placed on a slide and overlaid with a cover slip. The proportion of fluorescent cells was counted from 200 cells in a fluorescence microscope (Olympus $\mathrm{BH} 2$ with epifluorescence optics, Olympus Optical Co., Tokyo, Japan) using oil immersion and a fluorescein filter set.

The second plasma membrane viability test was performed using an automatic fluorometer (Fluoroscan Ascent, Thermo Electron Inc., Milford, MA, USA), which reads a 96-well microtitration tray and has an incubation compartment. The interference filter at the excitation path and that of the emission filter showed maximum transmission at $544 \mathrm{~nm}$ and $590 \mathrm{~nm}$, respectively. For the fluorometric assay, $20 \mathrm{mg}$ of PI was dissolved in $1 \mathrm{~L}$ of Beltsville Thawing Solution (BTS) (USDA, Beltsville, MD, USA) and dispensed in 3-mL aliquots. Equal aliquots (50 $\mu \mathrm{L})$ of BTS diluted semen sample $\left(80 \times 10^{6}\right.$ spermatozoa/ $\mathrm{mL}$ ) and PI solution were dispensed into a well and shaken gently for $2 \mathrm{~min}$. Spermatozoa from the same samples were killed by unprotected rapid freezing and slow thawing to obtain internal control samples consisting of only non- viable cells (100\% fluorescence). The control sample was immersed in liquid nitrogen for $1 \mathrm{~min}$ and thereafter allowed to stand at room temperature for $30 \mathrm{sec}$ and then $3 \mathrm{~min}$ in a water bath $\left(37^{\circ} \mathrm{C}\right)$. Blanks containing $50 \mu \mathrm{l}$ of diluted extender and $50 \mu \mathrm{l}$ of PI were analyzed separately for every experiment in 4 replicates; the incubation time was $8 \mathrm{~min}$. The percentage of fluorescence was calculated from the ratio of fluorescence intensities of the 
rapidly frozen control sample and the sample to be analyzed, after comparing with the blank values [22].

\section{Resazurin reduction test}

For the resazurin reduction test, $400 \mathrm{mg}$ of resazurin was dissolved in $1 \mathrm{~L}$ of distilled water. One part of this solution and 9 parts of $0.9 \% \mathrm{NaCl}$ were mixed [23]. An equal volume of this mixture and diluted sperm were combined and shaken for $2 \mathrm{~min}$, then incubated for $30 \mathrm{~min}$ at $34^{\circ} \mathrm{C}$ and measured with the fluorometer, using the same fluorometer settings as in the plasma membrane viability test.

\section{HOST}

For the HOST, semen was extended to $4 \times 10^{6}$ spermato$\mathrm{zoa} / \mathrm{mL}$. The hypo-osmotic solution was prepared by dissolving $1.352 \mathrm{~g}$ fructose and $0.735 \mathrm{~g} \mathrm{Na}$-citrate to distilled water (150 mOsm, pH 7.2). An aliquot of $0.125 \mathrm{~mL}$ of sperm was added to $0.5 \mathrm{~mL}$ of solution and the mixture was incubated for $30-45 \mathrm{~min}$ at $37^{\circ} \mathrm{C}$. A $5-\mu \mathrm{L}$ drop was placed on a slide and overlaid with a cover slip. A total of 200 spermatozoa per sample were evaluated for the presence of bent tails in light microscopy [24] and also analyzed with an automatic fluorometer. For fluorometric determination of the HOST, $0.5 \mathrm{~mL}$ of the same hypoosmotic solution (100 mOsmol $/ \mathrm{kg}$ ) were mixed with $0.125 \mathrm{~mL}$ of skim milk-extended semen (concentration $100 \times 10^{6}$ spermatozoa/mL). The fluorometric method was the same as for PI-stained semen. The mixture was incubated at $37^{\circ} \mathrm{C}$ and analyzed again after $3 \mathrm{~h}$.

\section{Morphology and bacteriology}

The frozen-thawed semen smears were air-dried and stained with Giemsa according to Watson [25]. A total of 100 spermatozoa were evaluated with light microscopy, magnification $\times 1250$, for major abnormalities (underdevelopment, acrosomal granules, other major acrosomal defects, diadem effects, tails bent under the head, dag effects, mid piece defects, and proximal droplets) and minor abnormalities (bent tail, twisted tail, loose normal heads, large heads, loose acrosomes, and mild acrosomal abnormalities) according to Blom [26].

The bacterial culture was performed by spreading a drop of each sample onto half a blood agar plate, using a $10-\mu \mathrm{L}$ sterile loop. After incubation for 24 and $48 \mathrm{~h}$ at $37^{\circ} \mathrm{C}$, colony forming units (CFUs) were counted and bacterial species recognized. If more than 100 CFUs were detected per sample, the number was not calculated further.

\section{Statistical methods}

Pearson and Spearman correlation coefficients were used to study the association between the parameters. The results were accounted for, if both correlation coefficients were congruent. P-values $<0.05$ were considered significant. The results were expressed as mean \pm the standard error of the mean (s.e.m.). The stallions were divided into 2 groups: foaling rate of mares $<60 \%$ or $>60 \%$. The independent sample t-test was used to test differences in the laboratory test parameters between the 2 groups of stallions. Statistical analysis was also performed separately from the material restricted to those stallions having $>20$ mares (19 stallions in Exp. 1 and 16 in Exp. 2).

\section{Results \\ Experiment I}

The percentage of normal spermatozoa varied from $51 \%$ to $89 \%$. Major abnormalities accounted for $9.5 \%$, including head abnormalities in $4.1 \%(1-12 \%)$, tail bent under the head $2.5 \%(0-6 \%)$, and mid piece defects $2.4 \%(0-$ $7 \%)$; minor abnormalities comprised $10.7 \%$ (3-31\%) including mainly bent tails $6.9 \%(1-29 \%)$, normal loose heads $1.4 \%(0-7 \%)$, and loose acrosomes $2 \%(0-5 \%)$. There was no association between morphological findings and foaling rate.

A total of $52 \%$ of the samples showed no microbial growth, in $41 \%<100$ CFUs per plate were detected, and in $7 \%>100$ CFUs per plate. The microbes were mainly coagulase-negative staphylococci or belonged to the families Enterococcus, Enterobacteriaceae, or Corynebacteriaceae. There was no association between bacteriological findings and foaling rate.

Average CASA motility and s.e.m. were as follows: TMOT $37.0 \pm 3.3$, PROG $27.6 \pm 2.7$, and VAP $58.7 \pm 2.0$; these values did not correlate with fertility. Average progressive motility evaluated in light microscopy showed following changes during incubation: 0 h $40.2 \pm 1.7$ ( $\min 10, \max$ $60), 1$ h $35.0 \pm 1.4(5,50), 2$ h $29.0 \pm 1.6(10,40), 3$ h 24.9 \pm 1.7 (10-40), and 4 h $21.0 \pm 1.7$ (5-40). Progressive motility correlated significantly with foaling rate after $2-4$ $\mathrm{h}$ of incubation (correlation coefficients $0.39-0.51$; $\mathrm{p}<$ 0.05 ). Stallions ( $>7$ mares) with foaling rates of $>60 \%$ appeared to retain sperm motility slightly better than stallions with foaling rates of $<60 \%$, although the difference was not statistically significant (Fig. 1). Similarly, semen resulting in foaling rates of $>60 \%$ showed higher plasma membrane integrity percentages measured with fluorometer than semen resulting in foaling rates of $<60 \%$, but the differences were not statistically significant (Fig. 2).

Sperm concentration and the total number of sperm in an AI dose showed huge variation: the average concentration \pm s.e.m. $383.2 \pm 48.6, \min 45, \max 1593$, and the average number of sperm/AI dose $713.2 \pm 47.2$, min 302, max 1777. When stallions having $>20$ mares were analyzed, the total number of sperm in an AI dose showed a significant negative correlation of 0.58 with foaling rate ( $\mathrm{p}<$ $0.05)$. The total number of sperm/AI dose and sperm concentration for stallion groups having foaling rates $<60 \%$ 


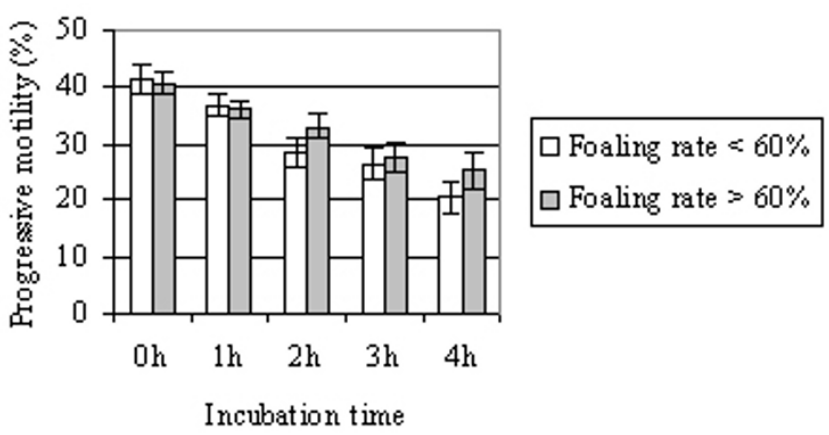

Figure I

(Exp. I) Mean ( \pm s.e.m.) progressive motility in light microscopy during 4-h incubation in stallion groups with foaling rates of $<60 \%$ or $>60 \%$. Number of mares per stallion was $>7$.

or $>60 \%$ are shown in Fig. 3, the difference being significant $(\mathrm{p}<0.05)$. For all other parameters correlation coefficients with fertility were low and non-significant.

When the various parameters were compared with each other, all motility parameters correlated significantly with each other (correlation coefficients varied from 0.44 to $0.81)$, similarly the plasma membrane integrity tests showed significant correlations between each other $(0.37$ - 0.83). CFDA/PI staining with light microscopy and with a fluorometer correlated significantly also with progressive motility before incubation. The total number of

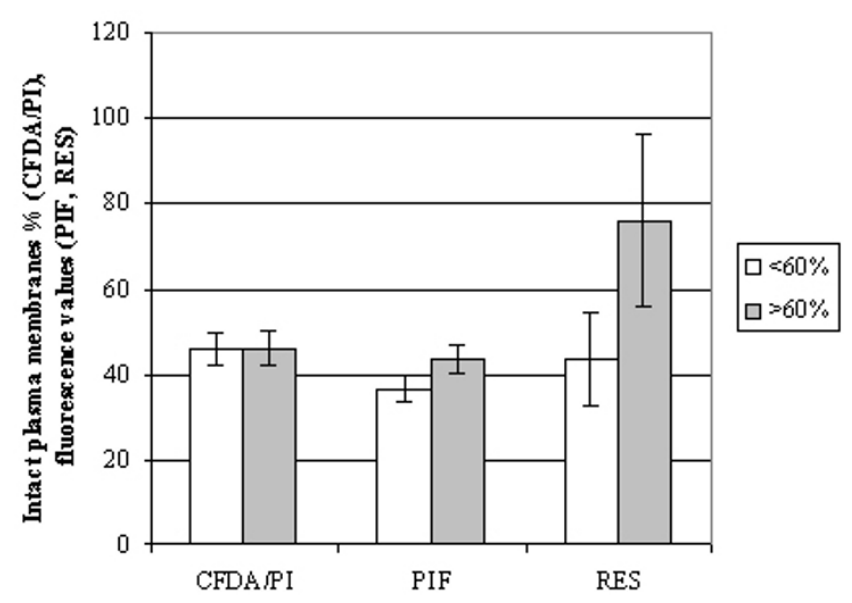

Figure 2

(Exp. I) Mean ( \pm s.e.m.) plasma membrane integrity parameters in stallion groups with foaling rates of $<60 \%$ or $>60 \%$. Number of mares per stallion was $>7$. CFDA/PI = plasma membrane integrity using light microscopy; PIF = plasma membrane integrity with PI staining using a fluorometer; RES = plasma membrane integrity with resazurin reduction test using a fluorometer.

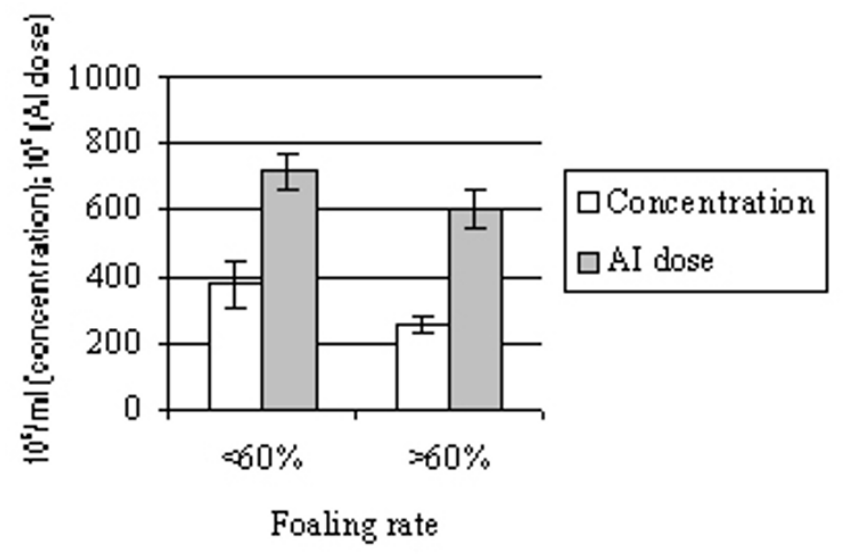

Figure 3

(Exp. I) Mean ( \pm s.e.m.) sperm concentration and total number of sperm in an Al dose in stallion groups with foaling rates of $<60 \%$ or $>60 \%$. Number of mares per stallion was $>7$.

sperm/AI dose showed a significant negative correlation with the other parameters, except with progressive motility during incubation (3-4 h) and CFDA/PI with light microscopy.

\section{Experiment 2}

The average HOST-values were $30.1 \pm 1.6$ (18-49) before incubation and $21.7 \pm 1.6(9-46)$ after $3 \mathrm{~h}$ of incubation. A significant correlation coefficient of -0.50 with foaling rate ( $\mathrm{p}<0.05$ ) was demonstrated before incubation. The average CFDA-values obtained in microscopy were $42.9 \pm$ 2.4 (14-66) before incubation and $33.0 \pm 1.8(12-48)$ after the 3 -h incubation. When stallions having $>20$ mares were analyzed, CFDA/PI staining with light microscopy at 0-h incubation and HOST with fluorometer after a 3-h incubation showed correlation coefficients of 0.5 with foaling rate $(\mathrm{p}>0.05)$. The HOST results in 2 stallion groups divided by their foaling rates are shown in Fig. 4. For other tests, correlation coefficients with foaling rate were low and non-significant. The TMOT and PROG values for stallions with foaling rates $<60 \%$ and $>60 \%$ are shown in Fig. 5.

When the various parameters were compared, TMOT, PROG, VAP, and RAP correlated after the $0-\mathrm{h}$ and 3-h incubations, correlation coefficients ranging from 0.5 to 0.8. CFDA, HOST and resazurin both by microscopy and fluorometer correlated after the 0 -h and 3-h incubations with coefficients of $0.4-0.8$, but no correlation was demonstrated between these parameters and parameters depicting motility. Before incubation, the concentration showed a significant negative correlation with CFDA/PI staining, using both light microscopy and the fluorome- 


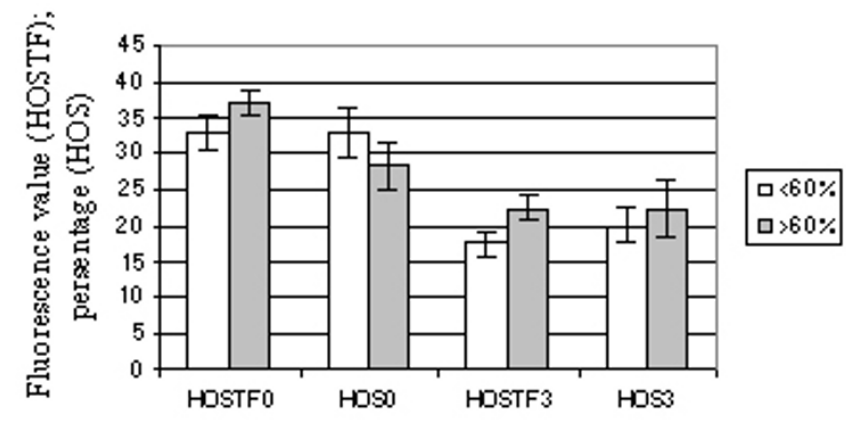

Figure 4

(Exp. 2) Mean ( \pm s.e.m.) percentage of sperm positive for hypo-osmotic swelling test (HOST) in stallion groups with foaling rates of $<60 \%$ or $>60 \%$. Number of mares per stallion was $>20$. HOSTFO $=$ HOST with fluorometer after $0-h$ incubation; HOSTF3 = HOST with fluorometer after 3-h incubation; HOSO = HOST with light microscopy after 0 -h incubation; HOS3 = HOST with light microscopy after 3-h incubation.

ter, but this correlation disappeared after the 3-h incubation.

\section{Discussion}

No single test was found to be a consistently reliable method for predicting the fertilizing capacity of semen. The results of Exp. 1 could not be repeated, although the stallions and methods used were partly similar. Differences in the outcome of these experiments could be

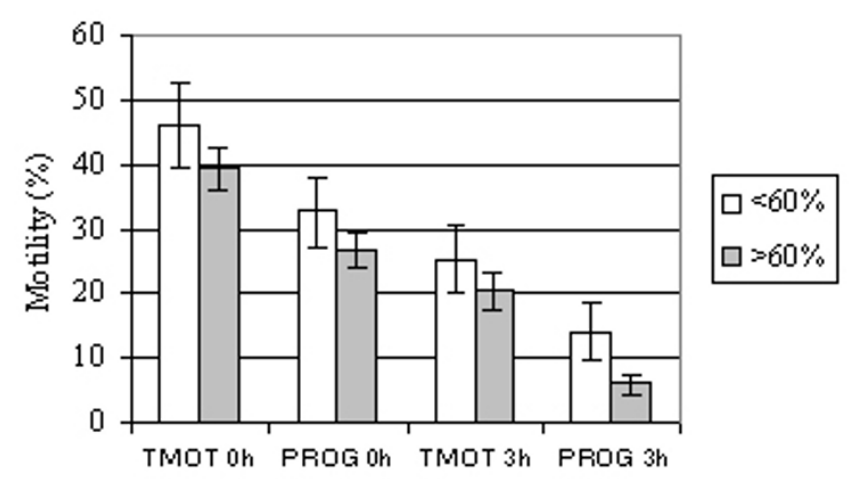

\section{Figure 5}

(Exp. 2) Mean ( \pm s.e.m.) total (TMOT) and progressive motility (PROG) immediately after thawing and after 3-h incubation in stallion groups with foaling rates of $<60 \%$ or $>60 \%$. Number of mares per stallion was $>7$.

explained also by different ejaculate batches.

\section{Plasma membrane integrity tests}

Some tendency for the plasma membrane integrity tests to be indicators of higher foaling rates was noted in our study. Fluorometers have not been much used in the examination of stallion semen. Although no strong correlation with foaling rate was found, the various fluorometric measurements did correlate with plasma membrane integrity in light microscopy and with each other. The use of fluorometers has some advantages in comparison to the use of light microscopy: it requires less time, and more cells in the sample are assessed [14]. In boars [27] and bulls [28], fluorometric measurements significantly correlated with fertility parameters.

Neild et al. [29] found no significant connection between the HOST and fertility but a tendency for the HOST to correlate with the number or services per pregnancy. We detected both negative and positive correlations, suggesting that this test is not suitable for evaluation of frozenthawed stallion semen.Nie et al. [30] reported lower pregnancy rates for HOS+ group. Blach et al. [12] found indirect evidence that many immotile spermatozoa possessed an intact plasma membrane, which would indicate that these two parameters do not correlate with each other. In our first experiment, plasma membrane integrity with light microscopy correlated with many other parameters, including motility. This is in disagreement with the study of Samper [13] who noted membrane integrity to show extremely poor correlation with motility, particularly in preserved semen. Likewise, in our second experiment correlation between motility and plasma membrane integrity was not observed.

\section{Motility}

Motility correlated with fertility in our first experiment but not in the second. One explanation can be pre-selection of semen. Most laboratories use $30 \%$ as a cut-off value when accepting semen for sale. The most important task of semen tests is to exclude semen of inferior quality, and this had already been done in the laboratories which had exported the semen. Another explanation is the use of different batches from several ejaculates from each stallion, but still the question on the accuracy of motility evaluation as a means of predicting fertility is raised. Semen from a stallion with the highest progressive motility $(60 \%)$ did not produce any pregnancies in 10 inseminated mares. Motility has been claimed by other investigators to be a poor predictor of pregnancy rates $[6,31]$. However, Voss and his coworkers [6] suggested that although the relationship between motility and fertility is poor in the stallion, spermatozoal motility and the quality of motility are still the most reliable estimates of fertility in practice. On the other hand, Jasko et al. [32] reported significant correlations between motility parameters and fertility.Newcombe [33] reported that pregnancies per 
insemination decreased when semen with low motility was used. VAP is not reliable or repeatable according to Kirk et al. [34]. The motility results immediately after thawing do not necessarily predict the results after incubation, as was seen with progressive motility in our first experiment. Longevity tests may therefore be useful in assessing viability of semen, although Voss et al. [6] suggested that longevity may be of limited value in predicting potential fertility. Despite these inconsistencies between studies, motility continues to have value as an easy and economical way for estimating relative cell health.

\section{Sperm numbers and concentration}

The total number of sperm/AI dose and concentration correlated negatively with many parameters. This can be explained by the tendency to increase AI dose and thus the concentration - the volume of one straw is limited - when low post-thaw motility is detected to increase the number of progressively motile spermatozoa and consequently the possibility of pregnancy [35]. This practice is only effective to a certain point; Amann [11] presented a doseresponse curve showing that fertility ceases to improve as the critical number of spermatozoa needed for maximum fertility of a given male has been reached. High doses and concentration may even decrease fertility. High sperm numbers and concentration provoked more intense inflammatory responses in the uterus $[36,37]$. Spermatozoa at higher AI doses arrived in the oviducts later than spermatozoa contained in smaller doses [37]. Fertility dropped when the AI dose contained $>900 \times 10^{6}$ frozen sperm [35].

\section{Morphology}

In our study, the percentage of morphologically normal semen and foaling rates did not correlate, perhaps because semen with a high percentage of morphological abnormalities is not frozen. Jasko et al. [38,32] showed the percentage of normal sperm to have a positive correlation with fertility. However, Voss et al. (1981) noted considerable variation in the morphologic characteristics among stallions and among ejaculates within stallions. They suggested that spermatozoal morphology may not be as valuable in evaluation of potential fertility in the stallion as it is in other large domestic animals.

\section{Problems of fertility studies in horses}

Problems confronted in previous fertility studies are also obvious here. The number of mares inseminated per stallion was small, the straws were not from the same batch, foaling rates were collected during several years - fertility of stallions can vary between years and decrease with age. Insemination conditions, veterinary skills, management of stud farms, criteria for mare selection, etc. vary considerably. Many other factors in addition to semen characteristics influence fertility. Although obtaining pregnancy rates is difficult, they are more accurate and reliable than foaling rates. However, this does not eliminate other factors affecting fertility, such as management and reproductive performance of mares, their previous reproductive status, and possible genetic factors [32]. A multi-center study by Samper et al. [35] summarized the major factors affecting pregnancy rates of mares bred with frozen semen: the technician, mare age and status, insemination volume, timing of insemination, and number of sperm per dose. Standardizing all these variables is clearly not possible.

Development of freezing methods for stallion semen is dependant on finding dependable correlations between laboratory tests and fertility, which appears very difficult to achieve since the results of different studies in this field tend to be contradictive. The present study was unable to address the question of which laboratory tests would accurately predict fertility of commercially produced stallion semen. Objectivity, repeatability, and accuracy are basic requirements for laboratory assays, but many semen analysis tests do not meet these requirements [7]. Quality control of cryopreserved stallion semen remains to be a problem in practice where e.g. flow cytometry is not available. For practical purposes, it would be most important to identify semen samples that are likely to have poor fertilizing potential [4]. Nie et al. [30] concluded that evaluating fresh spermatozoa offered no advantage for pregnancy over simply inseminating with spermatozoa not selected for any particular characteristics.

The constraints in horse breeding - small numbers of mares per ejaculate and per stallion and the tremendous variations in mare management and insemination - never allow us to carry out trials similar to what the cattle industry has done in developing freezing methods and AI techniques. Fertility trials of horses are bound to be of little value because of these reasons [16].

\section{References}

I. Colenbrander B, Gadella BM, Stout TAE: The predictive value of semen analysis in the evaluation of stallion fertility. Reprod Dom Anim 2003, 38:305-3II.

2. Boyle MS: Artificial insemination: assessing stallion semen quality after freezing. Equine Vet $J$ 1996, 28:5-6.

3. Tischner M: Evaluation of deep-frozen semen in stallions. $J$ Reprod Fertil 1979:58-59.

4. Graham JK: Assessment of sperm quality: a flow cytometric approach. Anim Reprod Sci 200I, 68:239-247.

5. Katila T: In vitro evaluation of frozen-thawed stallion semen: A review. Acta vet Scand 200I, 42:201-217.

6. Voss JL, Pickett BW, Squires EL: Stallion spermatozoal morphology and motility and their relationship to fertility. J Am Vet Med Assoc 198I, I 78:287-289.

7. Rodriguez-Martinez H: Laboratory semen assessment and prediction of fertility: still utopia? Reprod Dom Anim 2003, 38:312-318

8. Bataille $B$, Magistrini $M$, Palmer E: Analyse objective de la mobilite du sperme congele-decongele d'etalon. Essai de correlation avec la fertilite. (Objective determination of 
sperm motility in frozen-thawed stallion semen. Correlation with fertility). Anim Breed Abstr 1990:96-106.

9. Malmgren L: Sperm morphology in stallions in relation to fertility. Acta vet Scand 1992, 88:39-47.

10. Watson PF: Recent developments and concepts in the cryopreservation of spermatozoa and the assessment of their post-thawing function. Reprod Fertil Dev 1995, 7:87|-89|.

11. Amann RP: Can the fertility potential of a seminal sample be predicted accurately? J Andrology 1989, 10:89-98.

12. Blach EL, Amann RP, Bowen RA, Frantz D: Changes in quality of stallion spermatozoa during cryopreservation: plasma membrane integrity and motion characteristics. Theriogenology 1989, $31: 283-298$

13. Samper JC: Evaluation of cryopreserved semen: An alternative assay. Acta vet Scand 1992, 88:59-65.

14. Graham JK: Analysis of stallion semen and its relation to fertility. Vet Clin North Am Equine Pract 1996, I 2: I 9 - 129

15. Schweisguth DC, Hammerstedt RH: Evaluation of plasma membrane stability by detergent-induced rupture of osmotically swollen sperm. J Biochem Biophys Methods 1992, 24:8I-94.

16. Amann RP: Weaknesses in reports of "fertility" for horses and other species. Theriogenology 2005, 63:698-715.

17. De Albuquerque Lagares M: Bestimmung der osmotischen Resistenz von Hengstsamenzellen (The determination of the osmotic resistance of stallion spermatozoa). Thesis Hannover 1995.

18. Vidament M, Dupere AM, Julienne P, Evain A, Noue P, Palmer E: Equine frozen semen freezability and fertility field results. Theriogenology 1997, 48:907-917.

19. Kenney RM, Bergman RV, Cooper WL, Morse GW: Minimal contamination techniques for breeding mares: Techniques and preliminary findings. Proc Am Ass Equine Practnrs 1975, 21:327-336.

20. Varner DD, Scott DV, Johnson L: Use of a computerized system for evaluation of equine spermatozoal motility. Am J Vet Res 1991, 52:224-230.

21. Harrison RAP, Vickers SE: Use of fluorescent probes to assess membrane integrity of mammalian spermatozoa. J Reprod Fertil 1990, 88:343-352.

22. Alm K, Taponen J, Dahlbom M, Tuunainen E, Koskinen E, Andersson M: A novel automatized fluorometric assay to evaluate sperm viability and fertility in dairy bulls. Theriogenology 200I, 56:677-684.

23. Dart MG, Mesta J, Crenshaw C, Ericsson SA: Modified resazurin reduction test for determining the fertility potential of bovine spermatozoa. Arch Andr 1994, 33:71-75.

24. Jeyendran RS, Van der Ven HH, Perez-Pelaez M, Crabo BG, Zaneveld LJ: Development of an assay to assess the functional integrity of the human sperm membrane and its relationship to other semen characteristics. J Reprod Fertil 1984, 70:219-28.

25. Watson PF: Use of Giemsa stains to detect changes in acrosomes of frozen ram spermatozoa. Vet Rec 1975, 97:12-13.

26. Blom E: Sygelige tilstande i konsorganen og sperma som kassationsårsag ved import og eksport af avlstyre til og fra Danmark. (Pathological conditions in genital organs and sperm as a cause for the rejection of breeding bulls for import to and export from Denmark). Nord Vet Med 1983, 35:105-30.

27. Juonala T, Salonen E, Nurttila T, Andersson M: Three fluorescence methods for assessing boar sperm viability. Reprod Dom Anim 1999, 34:83-87.

28. Januskauskas A, Johannisson A, Rodriguez-Martinez H: Assessment of sperm quality through fluorometry and sperm chromatin structure assay in relation to field fertility of frozen-thawed semen from Swedish Al bulls. Theriogenology 200I, 55:947-96I.

29. Neild DM, Chaves MG, Flores M, Miragaya MH, Gonzalez E, Aquero $A$ : The HOS test and its relationship to fertility in the stallion. Andrologia 2000, 32:35I-3I5.

30. Nie GJ, Wenzel JGW, Johnson KE: Comparison of pregnancy outcome in mares among methods used to evaluate and select spermatozoa for insemination. Anim Reprod Sci 2002, 69:211-222.

31. Squires EL, Amann RP, Pickett BW: Preservation of stallion semen - Colorado experience. Symp Equine Reprod Denmark 1987:49-54.

32. Jasko DJ, Little TV, Lein DH, Foote RH: Comparison of spermatozoal movement and semen characteristics with fertility in stallions: 64 cases (1987-1988). I Am Vet Med Assoc 1992, 200:979-985

33. Newcombe JR: Evaluation of the fertilising capacity of frozenthawed horse semen. Vet Rec 1999, I45:46-47.

34. Kirk ES, Graham JK, Bruemmer JE, Squires EL: Evaluating frozen equine semen by flow cytometry. Anim Reprod Sci 200I, 68:348-349.

35. Samper JC, Vidament M, Katila T, Newcombe J, Estrada A, Sargeant J: Analysis of some factors associated with pregnancy rates of frozen semen: a multi-center study. Theriogenology 2002, 58:647-650.

36. Kotilainen T, Huhtinen M, Katila T: Sperm-induced leukocytosis in the equine uterus. Theriogenology 1994, 41:629-636.

37. Fiala SM, Pimentel CA, Mattos ALG, Amaral MG, Jobim MIM, Gregory RM, Mattos RC: Sperm transport and uterine inflammatory response after insemination in the mare. 15th Int Congr Anim Reprod Porto Seguro Brazil Abstracts 2004, 2:388.

38. Jasko DJ, Lein DH, Foote RH: Determination of the relationship between sperm morphologic classifications and fertility in stallions: 66 cases (1987-1988). J Am Vet Med Assoc 1990, 197:389-394.
Publish with Bio Med Central and every scientist can read your work free of charge

"BioMed Central will be the most significant development for disseminating the results of biomedical research in our lifetime. "

Sir Paul Nurse, Cancer Research UK

Your research papers will be:

- available free of charge to the entire biomedical community

- peer reviewed and published immediately upon acceptance

- cited in PubMed and archived on PubMed Central

- yours - you keep the copyright

Submit your manuscript here:

http://www.biomedcentral.com/info/publishing_adv.asp
BioMedcentral 